\title{
Evolving perspectives on mechanical circulatory support biocompatibility and interfaces
}

\author{
Marvin J. Slepian ${ }^{1,2}$, Joseph Italiano ${ }^{3}$, Danny Bluestein ${ }^{2}$, Jawaad Sheriff ${ }^{2}$, Yana Roka-Moiia ${ }^{1}$ \\ ${ }^{1}$ Deparment of Medicine and Biomedical Engineering, Sarver Heart Center, University of Arizona, Tucson, USA; ${ }^{2}$ Department of Biomedical \\ Engineering, Stony Brook University, Stony Brook, New York, USA; ${ }^{3}$ Brigham and Woman's Hospital, Harvard Medical School, Boston, \\ Massachusetts, USA \\ Correspondence to: Marvin J. Slepian, MD. Department of Medicine and Biomedical Engineering, Sarver Heart Center, University of Arizona, 1501 \\ North Campbell Avenue, Tucson, Arizona 85724, USA. Email: slepian@email.arizona.edu.
}

Submitted Dec 10, 2020. Accepted for publication May 09, 2021.

doi: 10.21037/acs-2020-cfmcs-247

View this article at: http://dx.doi.org/10.21037/acs-2020-cfmcs-247

Mechanical circulatory support (MCS) has significantly impacted the management and longevity of patients with advanced heart failure. Despite efficacy, MCS continues to be plagued by a seemingly constant group of adverse events. In recent years, while survival continues to improve and technology acceptance grows, we as a field, continue to gnaw away at adverse events. On a positive note, with newer ventricular assist device (VAD) designs, an apparent reduction in thrombotic complications has emerged. Though, to the cautious observer, a simultaneous shift to bleeding, rather than clotting, has occurred. This seems paradoxical, as one would expect with enhanced engineering of VAD designs and "removal" of thrombosis, one should be left with hemostasis, rather than with bleeding. Why are we now seeing this situation?

Generically, we are seeing this shift of adverse events as the underlying pathophysiology is complex, with many operative and, at times, countervailing processes ongoing simultaneously. New mechanisms of device-related platelet dysfunction and coagulopathy continue to be revealed, functioning on multiple scales-from the clinical to the molecular-with events and risks still present. It just depends on where you look, when you look, and at what scale. Herein we emphasize the complexity of these systems. As such, improvements in one aspect of an MCS system may appear to emerge clinically, though underlying pathophysiologic derangements may persist, leading to subclinical or clinical events in a different location, or at different times in another element of the system. Similarly, reduction in the propensity of one mechanistic process might permit other underlying processes to be revealed and dominate.
We outline and address several issues that must be kept in mind to allow our understanding of the pathophysiology of MCS to evolve.

\section{It's a system, not a device}

From a hemocompatibility perspective, an operative VAD is not a single device but a system, composed of inflow cannula, pump, outflow cannula and anastomoses to the ventricle and the outflow artery. Each of these components or zones is a site for local thrombogenicity, or activation for downstream thrombogenicity. While thrombosis of the actual pump may be reduced, risk remains for thrombus formation in the "free flow", or at other locations. This is heightened if component geometry is compromised and abnormal flows develop (1). Hence, if we look at a given zone or location, it may appear thrombus free. Yet, thrombosis and its consequences may be occurring distally. While this may not cause major clinical sequelae, it provides an underlying increased risk profile for pro-thrombosis, easily tipped in the setting of additive thrombotic drivers, i.e., infection.

\section{New "Opposing" mechanobiology mechanisms revealed}

The pathophysiology of underlying platelet biomechanics and blood-device interactions continues to be defined, providing insight that seemingly opposing processes may be ongoing concurrently (2). Recent evidence suggests that with increasing shear exposure, as occurs with repetitive 
cycles of VAD traverse, platelets are progressively damaged and impaired-manifested by decrease in size and count, mitochondrial exhaustion, downregulation of adhesion receptors, and reduced reactivity to biochemical agonists (3). These effects additively translate to platelet dysfunction favoring bleeding. When combined with acquired von Willebrand syndrome, dysfunctional angiogenesis and potent anti-thrombotic pharmacology, a shift to bleeding can occur (4). Interestingly, concurrently, mechanically damaged platelets readily shed microparticles, providing a catalytic surface for thrombin generation, facilitating thrombosis in the "free flow", widely distributing nidi of potential thrombosis systemically. In this setting of opposing processes, i.e., bleeding vs. clotting, with improved VAD design and reduced pump thrombosis, net bleeding dominates. This phenomenon of opposing "pushpull processes" is not unique, as precedent exists in other disease states such as; DIC, sepsis, massive traumatic injury and most recently, COVID-19 related coagulopathy.

\section{Biomarkers differentiating biochemical vs. shear-mediated platelet activation}

New biomarkers have emerged, valuable for discriminating the relative proportional effect of underlying concurrent processes. A distinct signature of biochemical vs. mechanical platelet activation has been defined (3). Increased levels of shear-biomarkers do correlate with MCS adverse clinical outcomes (5). These offer a tool for clinical decision making to guide therapeutic decisions to further reduce adverse events.

\section{Drugs alone are not the answer}

Current anti-thrombotic drugs have limited efficacy in highshear MCS conditions, frequently making matters worse. Aspirin has been shown to be ineffective in limiting SMPA at high shear within a VAD (6). This has been confirmed in several large clinical studies (7). The basic issue here is that current drugs do not target shear-sensitive mechanisms or pathways. On the horizon are new agents, which specifically address shear-sensitive cell mechanobiological targetsreferred to as "mechanoceuticals" (8). These agents operate predominantly via mechanical means, altering physical properties of platelets, i.e., stiffness, membrane fluidity and lipid composition. Targeting these holds promise in reducing platelet damage and activation by high shear, while preserving the "safety" of responsiveness to biochemical mediators.

\section{In silico methods and modeling can limit adverse events}

In silico methods for evaluating MCS hemocompatibility have increasingly been shown to be useful, preemptively reducing clinical events through advanced modeling approaches. Specifically, device thrombogenicity emulation (DTE), which combine in silico numerical simulations with in vitro measurements correlating device hemodynamics with platelet reactivity is effective in identifying "hot spots," i.e., zones of heightened propensity for disturbed flows, with high shear and high likelihood of SMPA and thrombus formation (9). DTE has been utilized effectively to improve device design and reduce thrombogenicity (10). It also is a valuable diagnostic method for identifying problematic regions responsible for VAD malfunction and clinical events.

\section{Virchow's Triad still is operative}

A dynamic balance between flow, surface, and inflammatory issues as driving forces of thrombosis, as suggested by Rudolf Virchow 165 years ago, with impact on bleeding as well, is always at play. Presently, while all of these constitutive elements are operative, we have limited means of determining the relative significance of any one element, at any given point in time, in a given patient. Developing means of monitoring these concurrent processes will ultimately be useful for guiding clinical operation, pharmacological management and to address emerging adverse events in complex MCS systems.

\section{Acknowledgments}

Funding: The study was supported by the National Institutes of Health grants U01 EB012487 (to DB, MJS, JS) and U01 HL131052 (to DB, MJS, JS), and by the Arizona Center for Accelerated Biomedical Innovation (ACABI) (to MJS, YRM).

\section{Footnotes}

Conflicts of Interest: The authors have no conflicts of interest to declare.

Ethical Statement: The authors are accountable for all aspects of the work in ensuring that questions related to the accuracy or integrity of any part of the work are appropriately investigated and resolved. 
Open Access Statement: This is an Open Access article distributed in accordance with the Creative Commons Attribution-NonCommercial-NoDerivs 4.0 International License (CC BY-NC-ND 4.0), which permits the noncommercial replication and distribution of the article with the strict proviso that no changes or edits are made and the original work is properly cited (including links to both the formal publication through the relevant DOI and the license). See: https://creativecommons.org/licenses/by-nc-nd/4.0/.

\section{References}

1. Chivukula VK, Beckman JA, Prisco AR, et al. Left Ventricular Assist Device Inflow Cannula Angle and Thrombosis Risk. Circ Heart Fail 2018;11:e004325.

2. Slepian MJ, Sheriff J, Hutchinson M, et al. Shear-mediated platelet activation in the free flow: Perspectives on the emerging spectrum of cell mechanobiological mechanisms mediating cardiovascular implant thrombosis. J Biomech 2017;50:20-5.

3. Roka-Moiia Y, Walk R, Palomares DE, et al. Platelet Activation via Shear Stress Exposure Induces a Differing Pattern of Biomarkers of Activation versus Biochemical Agonists. Thromb Haemost 2020;120:776-92.

4. Bartoli CR, Zhang DM, Hennessy-Strahs S, et al. Clinical and In Vitro Evidence That Left Ventricular Assist Device-Induced von Willebrand Factor Degradation

Cite this article as: Slepian MJ, Italiano J, Bluestein D, Sheriff J, Roka-Moiia Y. Evolving perspectives on mechanical circulatory support biocompatibility and interfaces. Ann Cardiothorac Surg 2021;10(3):396-398. doi: 10.21037/acs-2020cfmcs-247
Alters Angiogenesis. Circ Heart Fail 2018;11:e004638.

5. Consolo F, Sferrazza G, Motolone G, et al. Platelet activation is a preoperative risk factor for the development of thromboembolic complications in patients with continuous-flow left ventricular assist device. Eur J Heart Fail 2018;20:792-800.

6. Valerio L, Tran PL, Sheriff J, et al. Aspirin has limited ability to modulate shear-mediated platelet activation associated with elevated shear stress of ventricular assist devices. Thromb Res 2016;140:110-7.

7. Saeed O, Colombo PC, Mehra MR, et al. Effect of aspirin dose on hemocompatibility-related outcomes with a magnetically levitated left ventricular assist device: An analysis from the MOMENTUM 3 study. J Heart Lung Transplant 2020;39:518-25.

8. Available online: https://www.newswise.com/articles/ bioengineers-work-to-head-off-dangerous-blood-clots-inpatients-with-ventricular-assist-devices

9. Girdhar G, Xenos M, Alemu Y, et al. Device thrombogenicity emulation: a novel method for optimizing mechanical circulatory support device thromboresistance. PLoS One 2012;7:e32463.

10. Chiu WC, Tran PL, Khalpey Z, et al. Device Thrombogenicity Emulation: An In Silico Predictor of In Vitro and In Vivo Ventricular Assist Device Thrombogenicity. Sci Rep 2019;9:2946. 